\title{
Wireless Charger for Artificial Pacemaker
}

\author{
Abinaya.B ${ }^{\mathrm{a}, 1}$, Abirami.A.P $P^{\mathrm{b}}$, Divya.. $\mathrm{J}^{\mathrm{b}}$ and Rajalakshmi. $\mathrm{R}^{\mathrm{c}}$ \\ ${ }^{\mathrm{a}, \mathrm{b}}$ UG Scholar, Dept of ECE, Panimalar Engineering College, Chennai, India \\ ${ }^{\mathrm{c}}$ Associate Professor, Dept of ECE, Panimalar Engineering College, Chennai, India
}

\begin{abstract}
The vast majority of the modernized implantable devices and Biosensors are set inside a patient's body. To overcome this constraint, in this paper we have designed a rechargeable battery with wireless power transfer technique. The transdermal power transfer for the Pacemaker which is placed inside the heart should be possible by the concept of mutual inductance. The receiver loop ought to be situated inside the body and the transmitter curl ought to be situated outside of the body. The voltage controller will give or manage the necessary yield (output) voltage. The experiments were conducted on wireless charging through pork tissues reveal that from a $3.919-\mathrm{mw}$ power source, 3.072-mw power can be received at $300 \mathrm{kHz}$, reaching a high wireless power transfer efficiency of $78.4 \%$, showing that the charging is very fast. We have also connected a Bluetooth Module to the Atmega328 microcontroller. This Bluetooth technology is used in the Android mobile application to notice the charging levels of the pacemaker. This Inductive power transfer technique takes out the danger of contamination which is brought about by the medical procedure.
\end{abstract}

Keywords: Microcontroller, Pulse-sensor, Mutual Induction, wireless charging.

\section{Introduction}

The implantable cardiac pacemakers have been playing a very important role in treating a wide range of cardiac disorders and have been recognized as life savers for the patients with cardio vascular diseases. In case of any absence of intrinsic impulses, the pacemaker stimulates the heart. Millions of people around the world have benefited from the systems such as implantable cardiac pacemakers. These systems are commonly used to treat patients suffering from arrhythmia - a disease caused due to the abnormal activity of the heartbeat. Nonetheless, there is an enormous limitation regarding the power supply. At present, the Bio-implantable pacemakers were powered using the disposable lithium-iodine batteries. Since the batteries used for these pacemakers are disposable in nature, they just last from 5 to 10 years or a normal of seven years. Once, the pacemaker battery is depleted completely, it is prudent to supplant the pacemaker with a fresher one, which can be done by doing a medical procedure. To beat this limitation, we have proposed wireless power transmission method using Inductive coupling. This method also avoids the risks like infections and allergies caused in the elderly people as a result of performing a surgery.

\footnotetext{
${ }^{1}$ Rajalakshmi.R, Associate professor/ECE, Panimalar Engineering College, Chennai, India. Email:rajeeramanathan@gmail.com
} 


\section{Existing System}

The current implantable pacemaker is controlled with lithium-iodine battery, which can last from 5 to 10 years or more - on the normal around seven years, now often weightless than $30 \mathrm{~g}$. They are usually implanted subcutaneously in the intraclavicular area. The energy which is needed for the activity of hardware of the pacemaker is given by this battery, which incorporates the control, detecting and heartbeat producing units. The current drain depends on the electrode types, pulse generation types and also the circuitry of the pacemaker. As the life span of a heart pacemaker implies its battery life, it is fundamental to have the hardware to distinguish the left-over existence of the battery in a basic and dependable way.

\subsection{Literature Review}

We have surveyed these papers which are centered on the wireless power transfer techniques for biomedical applications. These papers were arranged with in the descending order supported the year they were published.

Table 1. Literature Review

\begin{tabular}{|c|c|c|c|c|}
\hline S.no & Journal & Year & Authors & Findings \\
\hline 1 & $\begin{array}{l}\text { IEEE Miniaturized } \\
\text { wireless power } \\
\text { transfer system for } \\
\text { neuro stimulation } \\
{[1]}\end{array}$ & 2020 & $\begin{array}{l}\text { Gian Luca Barbruni, } \\
\text { PaoloMottoRos, } \\
\text { DaniloDemarchi, } \\
\text { SandroCarrara, } \\
\text { Diego Ghezzi [1] }\end{array}$ & $\begin{array}{l}\text { Compares and uniquely selects } \\
\text { the most suitable wireless } \\
\text { power transfer method for } \\
\text { neuro stimulation with } \\
\text { minimally invasive } \\
\text { miniaturized implants [1]. }\end{array}$ \\
\hline 2 & $\begin{array}{l}\text { IEEE Bio integrated } \\
\text { and wirelessly } \\
\text { powered } \\
\text { implantable brain } \\
\text { devices [2] }\end{array}$ & 2020 & $\begin{array}{l}\text { Rupam Das, } \\
\text { FarshadMoradi, } \\
\text { HadiHeidari [2] }\end{array}$ & $\begin{array}{l}\text { Designed a system for bio- } \\
\text { integrated and wirelessly } \\
\text { powered Implantable neural } \\
\text { devices in animals aimed at } \\
\text { long-term neural interfacing } \\
\text { [9]. }\end{array}$ \\
\hline 3 & $\begin{array}{l}\text { IEEE Wireless } \\
\text { power receiver } \\
\text { with wide } \\
\text { dynamic range for } \\
\text { bio-medical } \\
\text { implants [10] }\end{array}$ & 2019 & $\begin{array}{l}\text { Hankyu Lee, } \\
\text { Seungchul Jung, } \\
\text { YeunheeHuh, } \\
\text { SangJoonKim [10] }\end{array}$ & $\begin{array}{l}\text { Proposes a wireless power } \\
\text { receiver with wide input power } \\
\text { dynamic range and includes a } \\
\text { Dickson-charge pump for low } \\
\text { power region and active } \\
\text { rectifier for high efficiency at } \\
\text { high power region [3]. }\end{array}$ \\
\hline
\end{tabular}

4 IEEE Practical

Inductive link

design for

Biomedical

Wireless Power 2018

Matthew

Schormans,

VirgilioValente,

Transfer [4]

Andreas

Demosthenous [4]

Proposed a method which reviews the equations that are required to design an inductive link for biomedical wireless power transfer, with a focus on practicality [11]. 
IEEE A Wireless

Charging Circuit with high power efficiency and security for implantable devices [12]

$\begin{array}{lr}\text { IEEE } & \text { Optimal } \\ \text { Design } & \text { of } \\ \text { Wireless } & \text { Power }\end{array}$ Transmission Link for millimetersized Biomedical Implants [6]

7 IEEE Wireless Power Systems for charge super capacitors as power sources for implantable devices [7]

IEEE Bluetooth based low energy Qi compliant battery charger for implantable medical devices [8]

\begin{abstract}
Aims at inductively coupled implantable medical applications with wide range input-voltage and light output load along with a state checking operation ensures the security of the whole system [5].
\end{abstract}

YaoLu,HanjunJiang, SongpingMai,Zhihu aWang [12]

Developed a method which presents a design methodology for millimeter sized implantable DukjuAhn, biomedical devices. Power MaysamGhovanloo Transfer Efficiency is [6] maximized because of the optimal operating frequency found.

Presents a wireless power charging system for storage energy in supercapacitor as a power source for bio-medical
Pablo Aqueveque, JulianoBarboza [7] implants by using a class $\mathrm{E}$ amplifier to give voltage to an inductive link and storage energy in a supercapacitor [7].

SamiHached, Areftrigui, ImaneElKhalouffi, Presents a smart battery charging system implementing the Qi wireless power transmission standard. MohamadSawan, OlegLoutochin, JacquesCorcos [8]
Proposed device offers the possibility of charging batteries with any Qi certified power transmitter [8]

\section{Proposed System}

To overcome the limitations, present in the disposable lithium-ion batteries, wireless power transmission method using inductive coupling is proposed. coupling deals with the guideline of attractive field acceptance between two loops. Here, essential loop is set external the human body, which goes about as remote transmitter, though auxiliary curl is set inside the body close to the heart which goes about as a remote beneficiary. Essential curl of the energy transmitter creates a fluctuating attractive field across the optional loop of the energy recipient inside the field.

\subsection{Voltage Regulators}

The LM78XX (Voltage regulator) arrangement of three terminal controllers is accessible with many fixed yield voltages which makes them helpful in a different scope of utilizations. The $12 \mathrm{v} / 1 \mathrm{~A}$ lead-acid battery connected to the wireless receiver gets charged from the receiver as a result of mutual induction. Additionally, a pulse sensor is added to the battery charging circuit. 

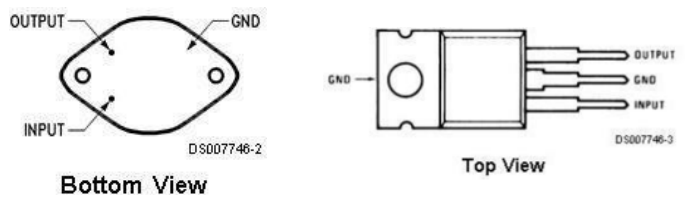

Figure 1.Source: Google

\subsection{Pulse Sensor}

Pulse sensor is a small electronic device that consists of pulse generator and sensing unit for sensing the electrical activity of the heart and provides electrical stimuli in order to trigger cardiac contraction if the heart rate is abnormal. The pacemaker is in "fixed pacing mode" by default while placing it over patient's body. When it is in an "operating mode" a time interval in between the pulses are measured that provides the battery energy which is helpful in predicting life expectancy of devices. A real time long term and continuously monitoring the activity of pacemaker is desirable.

\subsection{Bluetooth Module}

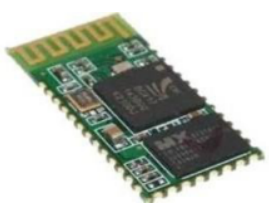

Figure 2. Source: Google

For transparent wireless communication setup, a Serial Port Protocol is designed. It's a Bluetooth module that has radio transceiver and base band of $2.4 \mathrm{GHz}$ along with the modulation rate of $3 \mathrm{Mbps}$. These components are connected to the Atmega328 microcontroller. This is safe for human tissues and electromagnetic compatible with the cardiac pacemaker.

\section{Result and Conclusions}

In our paper, a wireless charger for artificial pacemaker is explained by an approach of inductively coupling wireless transmission technique. This enables one to achieve an optimized coil design after taking tissues properties and the device oriented into account. The total time to charge the battery is very fast as a result of high wire-less power transfer efficiency of $78.4 \%$. This paper provides a new vision to people for charging a pacemaker which is embedded in one's body without many hazards, and it will also be cost effective. Hence, this method of approach is one of the cheapest solutions which might be easy to implement in future pacemaker and it will also increase user's satisfaction based on their requirements. 


\section{References}

[1] Gian Luca Barbruni, Paolo Motto Ros, Danilo Demarchi, Sandro Carrara, Diego Ghezzi. Miniaturised Wireless Power Transfer Systems for Neuro stimulation: A Review, IEEE Transactions on Biomedical Circuits and Systems, 2020

[2] RupamDas, Farshad Moradi, Hadi Heidari., Bio integrated and wirelessly powered implantable brain devices.IEEE Transactions on Biomedical circuitsandsystems, 2020

[3] Hankyu Lee, Seungchul Jung, Yeunhee Huh, Sang JoonKim. Wireless Power Receiver with Wide Dynamic Range for Biomedical Implants,2019 IEEE Wireless Power Transfer Conference (WPTC), 2019

[4] Matthew Schormans, Virgilio Valente, Andreas Demosthenous. Correction to Practical Inductive Link Design for Biomedical Wireless Power Transfer: A Tutorial [Oct 18 1112-1130]", IEEE Transactions on Biomedical Circuits and Systems,2019

[5] Yao Lu, Hanjun Jiang, Songping Mai, Zhihua Wang.A Wireless Charging Circuit with high powe efficiency and security for implantable devices", 2016 IEEE Biomedical Circuits and Systems Conference (BioCAS), 2016

[6] Dukju Ahn, Maysam Ghovanloo, Optimal Design of Wireless Power Transmission Links for Millimeter-Sized Biomedical Implants, IEEE Transactions on Biomedical CircuitsandSystems, 2016

[7] Pablo Aqueveque, Juliano Barboza, Wireless power system for charge super capacitors as powersources for implantable devices, 2015 IEEE PELS Workshop on Emerging Technologies: WirelessPower (2015 WoW), 2015

[8] SamiHached, ArefTrigui, Imane ElKhalouffi, Mohamad Sawan, Oleg Loutochin, Jacques Corcos.A Bluetooth-based Low-Energy Qi-compliant battery charger for implantable medical devices .2014 IEEE International Symposium on Bioelectronics and Bioinformatics (IEEE ISBB 2014), 2014

[9] Internet Source - eprints.gla.ac.uk

[10] Internet Source - www.wpw2019.org

[11] Internet Source - repository.tudelft.nl

[12] Internet Source- researchr.org 